\title{
Cardioprotective effects and mechanism of Radix Salviae miltiorrhizae and Lignum Dalbergiae odoriferae on rat myocardial ischemia/reperfusion injury
}

\author{
FEI MU ${ }^{1 *}$, JIALIN DUAN ${ }^{1 *}$, HAIXU BIAN ${ }^{1}$, YING YIN $^{1}$, YANRONG ZHU $^{1}$, GUO WEI $^{1}$, YUE GUAN $^{1}$, \\ YANHUA WANG ${ }^{1}, \mathrm{CHAO} \mathrm{GUO}^{1}$, AIDONG WEN ${ }^{1}$, YONG YANG $^{2}$ and MIAOMIAO XI ${ }^{1}$ \\ ${ }^{1}$ Department of Pharmacy, Xijing Hospital, Fourth Military Medical University, Xi'an, Shaanxi 710032; ${ }^{2}$ Department of \\ Pharmacy, Sichuan Academy of Medical Sciences and Sichuan Provincial People's Hospital, \\ Chengdu, Sichuan 610072, P.R. China
}

Received June 15, 2016; Accepted April 25, 2017

DOI: $10.3892 / \mathrm{mmr} .2017 .6821$

\begin{abstract}
Radix Salviae miltiorrhizae (SM) and Lignum Dalbergiae odoriferae (DO) are traditional Chinese medicinal herbs used to treat ischemic heart disease and other cardiovascular diseases; however, to the best of our knowledge, there are currently few studies regarding their effects. The present study aimed to investigate the cardioprotective effects of SM and DO during myocardial ischemia/reperfusion (MI/R) injury in rats, and explore the molecular mechanisms that underlie their actions. In the present study, Sprague-Dawley rats were pretreated with SM, the aqueous extract of DO (DOA) and the volatile oil of DO (DOO), either as a monotherapy or in combination for 7 days. Subsequently, the rats were subjected to $30 \mathrm{~min}$ of ischemia followed by $180 \mathrm{~min}$
\end{abstract}

Correspondence to: Professor Yong Yang, Department of Pharmacy, Sichuan Academy of Medical Sciences and Sichuan Provincial People's Hospital, 32 West Second Section First Ring Road, Chengdu, Sichuan 610072, P.R. China

E-mail: yyxpower@163.com

Professor Miaomiao Xi, Department of Pharmacy, Xijing Hospital, Fourth Military Medical University, 127 Changle West Road, Xi'an, Shaanxi 710032, P.R. China

E-mail: miaomiaoxi2014@163.com

*Contributed equally

Abbreviations: $\mathrm{MI} / \mathrm{R}$, myocardial ischemia/reperfusion; CK-MB, creatine kinase-MB; LDH, lactate dehydrogenase; SOD, superoxide dismutase; MDA, malondialdehyde; TNF- $\alpha$, tumor necrosis factor- $\alpha$; IL-6, interleukin-6; TTC, 2,3,5-triphenyltetrazolium chloride; TUNEL, terminal deoxynucleotidyl transferase-mediated dUTP nick end-labeling

Key words: myocardial ischemia/reperfusion injury, Radix Salviae miltiorrhizae, Lignum Dalbergiae odoriferae, effective treatment durations, cardioprotection, metabonomics of reperfusion. Traditional pharmacodynamic evaluation and metabonomics based on gas chromatography/time-of-flight mass spectrometry were used to identify the therapeutic effects of these traditional Chinese medicines. The results revealed that SM, DOA and DOO monotherapies ameliorated cardiac function, and this effect was strengthened further when used in combined therapies. Among the combined treatments, SM + DOO exhibited the greatest potential $(\mathrm{P}<0.05)$ to improve electrocardiogram results and heart rate, reduce the heart weight index and myocardial infarct size, and decrease the levels of creatine kinase-MB and lactate dehydrogenase. In addition, metabonomics-based findings, including the principal component analysis and partial least squares discriminant analysis score plot of the metabolic state in rat serum, provided confirmation for the aforementioned results, verifying that $\mathrm{SM}+\mathrm{DOO}$ exerted synergistic therapeutic efficacies to exhibit a greater effect on rats with $\mathrm{MI} / \mathrm{R}$ injury when compared with the other pretreatment groups. Furthermore, the most effective duration of SM + DOO treatment was $30 \mathrm{~min}$ and the least effective duration was $180 \mathrm{~min}$. Treatment with $\mathrm{SM}+\mathrm{DOO}$ also significantly $(\mathrm{P}<0.01)$ reduced the number of terminal deoxynucleotidyl transferase-mediated dUTP nick end-labeling-positive cells, tumor necrosis factor- $\alpha$ andinterleukin- 6 expression, and malondialdehyde content, and increased the serum and tissue activity of superoxide dismutase. These results indicated that the combined effects of SM + DOO may be more effective compared with the single pretreatments against MI/R injury in rats. This effect may be achieved partly through anti-apoptotic, antioxidant and anti-inflammatory activities. Therefore, SM + DOO may be considered an effective and promising novel strategy for the prophylaxis and treatment of ischemic heart disease.

\section{Introduction}

Ischemic heart disease (IHD) is a global public health concern due to its rising incidence, and the resulting high levels of morbidity and mortality $(1,2)$. The prevention and management of myocardial ischemia/reperfusion (MI/R) injury is essential following surgery for coronary heart disease (3); 
however, it remains a major clinical issue associated with IHD treatment (4). The outcomes of MI/R injury include reperfusion arrhythmias, myocardial stunning, myocardial hibernation and myocardial dysfunction (5). It has previously been reported that diverse pathological factors, including inflammation, oxidative stress and apoptosis, are involved in the pathological mechanisms underlying MI/R injury (6-10). Therefore, multitarget therapeutic strategies for MI/R injury are urgently required.

Traditional Chinese medicine (TCM) has been applied in humans for thousands of years, and has generated an increasing interest for the mitigation of MI/R injury due to numerous therapeutic effects $(11,12)$. Radix Salviae miltiorrhizae (SM; Chinese name, Danshen) and Lignum Dalbergiae odoriferae (DO; Chinese name, Jiangxiang) are TCM herbs widely used in China, Korea and Japan for the treatment of IHD and other cardiovascular diseases $(13,14)$. In clinical practice, SM and DO are commonly used in combination, the most well-known treatments including Guanxin II, which contains SM and the aqueous extract of DO (DOA), the QiShenYiQi drop pill and the Guanxin Danshen tablet, which contains SM and the volatile oil of DO (DOO). Therefore, it is necessary to investigate the therapeutic effects of different combinations, and to elucidate why and how the combination of SM and DOO considerably outperforms individual herbal treatments in cardioprotection.

Metabonomics is a novel scientific platform that may be used to determine the integrity and systemic features of TCM, which usually involves multicomponent, multipathway and multitarget treatments (15). As a powerful analytical platform, the application of metabonomics has markedly increased in the fields of pharmaceutical discovery and development (16), evaluation of drug efficacy and toxicity $(17,18)$, and in novel therapy and potential biomarker discovery $(19,20)$. Numerous analytical tools have been employed, including ${ }^{1} \mathrm{H}$-nuclear magnetic resonance spectroscopy, high-performance liquid chromatography-mass spectrometry (HPLC-MS), capillary electrophoresis-MS and gas chromatography (GC)-MS (21-24). GC-MS is advantageous over the other techniques in the field of metabonomics, due to its numerous structural databases (25), high sensitivity and reproducibility. It is thought to be vital in the future of metabonomics research.

In the present study, SM and DO, alone or in combination, were orally administered to rats prior to $\mathrm{MI} / \mathrm{R}$ injury in order to investigate their effects and potential underlying mechanisms. The present study performed a metabonomics assay using GC/time-of-flight (TOF)-MS and traditional pharmacodynamic evaluation, which may provide novel strategies for the assessment of the holistic efficacy and synergism of TCM. In addition, the present study aimed to elucidate the most and least effective durations of the various treatments during reperfusion.

\section{Materials and methods}

Drugs and reagents. The SM extract was purchased from Xi'an Honson Biotechnology Co.,Ltd.(batch no. 150110; Xi'an,China) and the major components were evaluated by HPLC analysis, as previously described $(26,27)$. Briefly, the components were analyzed on an Agilent TC-C18 column (4.6x250 mm, $5 \mu \mathrm{m}$ particle size; Agilent Technologies, Inc., Santa Clara, CA,
USA), using a gradient elution of acetonitrile $/ 0.2 \%$ aqueous formic acid as the mobile phase at a flow rate $0.8 \mathrm{ml} / \mathrm{min}$ and $\mathrm{UV}$ detection at $270 \mathrm{~nm}$. The column temperature was $30^{\circ} \mathrm{C}$ with a $20 \mu \mathrm{l}$ injection volume. The major components of SM are hydrophobic tanshinones and hydrophilic salvianolic acids. DO, of which the major active components are flavonoids and volatile oil, was purchased from Xi'an Xiaocao Botanical Development Co., Ltd. (batch no. XC20150302; Xi'an, China). Evans blue and 2,3,5-triphenyltetrazolium chloride (TTC) were purchased from Sigma-Aldrich (Merck KGaA, Darmstadt, Germany). Lactate dehydrogenase (LDH; cat no. A020-2), superoxide dismutase (SOD; cat no. A003-1) and malondialdehyde (MDA; cat no. A003-1) test kits were purchased from Nanjing Jiancheng Bioengineering Institute (Nanjing, China). Creatine kinase-MB (CK-MB; cat no. F15213), tumor necrosis factor- $\alpha$ (TNF- $\alpha$; cat no. F16960) and interleukin-6 (IL-6; cat no. F15870) enzyme-linked immunosorbent assay (ELISA) kits were purchased from Xitang Biology Technology Company (Shanghai, China).

Herbal extraction. SM was dried to a constant weight at $50^{\circ} \mathrm{C}$ and ground into powder ( $<1 \mathrm{~mm})$. Subsequently, $150 \mathrm{~g} \mathrm{SM}$ was immersed with 8 -fold the volume of water for $30 \mathrm{~min}$ and refluxed twice $(1.5 \mathrm{~h} /$ reflux). The resulting suspension was filtered, concentrated to $75 \mathrm{ml}$ using a rotary evaporator, and stored at $4^{\circ} \mathrm{C}$. Using the same procedure, DOA was prepared and DOO was isolated by steam distillation for $5 \mathrm{~h}$ with a yield of $0.5 \%$ DOO, which was then stored in glass bottles at $4^{\circ} \mathrm{C}$ for future analysis.

Animals and MI/R injury model. Sprague-Dawley male rats (n=78; age, $\sim 2$ months; weight, $280 \pm 20$ g) were obtained from the Laboratory Animal Center of the Academy of Fourth Military Medical University [Xi'an, China; qualified production number, SCXK-(Jun)-2012-007]. Rats were housed in cages at a temperature of $24 \pm 2{ }^{\circ} \mathrm{C}$ and relative humidity of $45 \pm 15 \%$, under a 12 -h light/dark cycle, with free access to food and water. All protocols were performed in accordance with the guidelines established by the Guide for the Care and Use of Laboratory Animals of the Fourth Military Medical University, and the study was approved by the Laboratory Animal Management and Ethics Committee of the Stomatology Hospital of The Fourth Military Medical University. Prior to surgery, rats in the Sham and Model groups ( $n=6 /$ group) were intragastrically pretreated with purified water, whereas rats in the treatment groups were intragastrically administered the indicated drugs for 7 days. Following the last drug pretreatment, the surgical protocol was performed and the MI/R injury model was established in all rats, with the exception of rats in the Sham group, by direct coronary ligation as previously described (28). Briefly, Sprague-Dawley rats were intubated and artificially ventilated with a rodent ventilator (HX-100E; Chengdu Taimeng Technology Co., Ltd., Chengdu, China) under anesthesia with $10 \%$ chloral hydrate (3 $\mathrm{ml} / \mathrm{kg}$, intraperitoneally). Anormal electrocardiogram (ECG) was recorded following subcutaneous placement of electrodes and connection to an electrocardiograph (BL-420S; Chengdu Taimeng Technology Co., Ltd.). Coronary artery ligation was achieved with a plastic snare fixed onto the left anterior descending (LAD) coronary artery. A 6-0 silk suture 
was passed underneath the LAD (2-3 mm inferior to the left auricle) and tied. Following $30 \mathrm{~min}$ of ischemia, the plastic snare was removed and the myocardium was reperfused for $180 \mathrm{~min}$. The Sham group underwent thoracotomy and cardiac exposure without coronary ligation.

Experimental protocol. The dosages of SM and DO applied were based on our previous results and those of others $(14,29)$. Different combinations of SM $5 \mathrm{~g} / \mathrm{kg}$, DOA $2.5 \mathrm{~g} / \mathrm{kg}$ and DOO $0.5 \mathrm{ml} / \mathrm{kg}$ were used to evaluate their protective effects on $\mathrm{MI} / \mathrm{R}$ injury.

Firstly, rats were randomized into the following eight groups (n=6 rats/group): Sham, Model, Diltiazem (16 mg/kg/day; positive control group; Tianjin Tianbian Pharmaceutical Co., Ltd., Tianjin, China), SM (5 g/kg/day), DOA (2.5 g/kg/day), DOO (0.5 ml/kg/day), SM+DOA ( $5+2.5 \mathrm{~g} / \mathrm{kg} /$ day, respectively) and $\mathrm{SM}+\mathrm{DOO}(5 \mathrm{~g} / \mathrm{kg} /$ day $+0.5 \mathrm{ml} / \mathrm{kg} /$ day, respectively). The ECG and heart rate were monitored and recorded continuously; following $180 \mathrm{~min}$ of reperfusion, serum samples were collected from the right carotid artery and used to determine the levels of myocardial marker enzymes and to perform the metabonomics study. Subsequently, rats were sacrificed and the hearts were harvested to assess infarct size and heart weight index (HWI; HWI = heart weight/body weight $\mathrm{x} 100 \%)$. The variation in heart rate was calculated as follows: Heart rate variation $=$ (heart rate at end of reperfusion $)$ - (heart rate before surgery).

In order to elucidate the most and least effective treatment durations of these herbal medicines, the rats were randomized into five groups ( $\mathrm{n}=6$ rats/group): Sham, Model, SM, DOO and $\mathrm{SM}+$ DOO. The most and least effective treatment durations were determined by measuring the indicators associated with myocardial injury: CK-MB and LDH, at various time-points of reperfusion $(5,15,30,45,60,120$ and $180 \mathrm{~min})$ by taking blood samples from the right carotid artery.

In order to investigate the mechanism underlying the action of SM + DOO, following180 min of reperfusion, serum samples were collected from rats in the Sham, Model, SM, $\mathrm{DOO}$ and $\mathrm{SM}+\mathrm{DOO}$ groups, rats were sacrificed, and hearts were harvested and stored at $-80^{\circ} \mathrm{C}$ for further biochemical analysis. The focus of this investigation was mainly on the three mechanisms associated with apoptosis, oxidative stress and inflammation.

Measurement of myocardial infarct size. At the end of the reperfusion period, the coronary artery was occluded and 3\% Evans blue $(2 \mathrm{ml})$ was perfused into the aorta and coronary arteries. Subsequently, rats were sacrificed and the entire ventricular tissue was sliced into five $\sim 2 \mathrm{~mm}$-thick sections through the transverse axis from the apex to the atrioventricular groove. Tissues were incubated in $3 \mathrm{ml} 2 \%$ TTC at $37^{\circ} \mathrm{C}$ for $15 \mathrm{~min}$ and all slices were then fixed with $4 \%$ paraformaldehyde overnight at room temperature, as previously described (30). Images of each slice were captured using a Canon Digital IXUS 980 IS digital camera (Canon, Inc., Tokyo, Japan) and analyzed. The infarct area was pale white, whereas the non-infarct, but at risk, area was red. The total area at risk (AAR) was indicated by white and red staining. The myocardial infarct size was measured and expressed as a percentage of infarct size over AAR, which was calculated by computerized planimetry using Image-Pro Plus software version 6.0 (Media Cybernetics, Inc., Rockville, MD, USA).

Assessment of myocardial marker enzymes. Blood samples from the right carotid artery of rats were collected following $180 \mathrm{~min}$ of reperfusion. Samples were maintained for $30 \mathrm{~min}$ at room temperature and the serum was separated by centrifugation at 2,258 $\mathrm{x}$ g for $15 \mathrm{~min}$ at $4^{\circ} \mathrm{C}$ (TDZ4A-WS; Shanghai Lu Xiangyi Centrifuge Instruments Co., Ltd., Shanghai, China). The biochemical markers of myocardial ischemic injury, CK-MB and LDH, were detected in serum. All measurements were performed according to the kit manufacturers' protocols.

Sample preparation and serum metabonomics. Each $50 \mu 1$ serum sample underwent extraction procedures with $0.3 \mathrm{ml}$ methanol and $20 \mu \mathrm{l} \mathrm{L}$-2-chlorophenylalanine $(1 \mathrm{mg} / \mathrm{ml}$ stock in $\mathrm{dH}_{2} \mathrm{O}$ ), which was used as an internal standard. All mixtures were vortex-mixed at a high speed for $5 \mathrm{~min}$, followed by centrifugation at $7,740 \mathrm{x}$ g for $15 \mathrm{~min}$ at $4^{\circ} \mathrm{C}$. The supernatant $(\sim 0.4 \mathrm{ml})$ was then transferred to a new $2 \mathrm{ml} \mathrm{GC} / \mathrm{MS}$ glass vial. In addition, an equal volume of $\sim 11 \mu \mathrm{l}$ was taken from each sample and was transferred into a new $2 \mathrm{ml} \mathrm{GC} / \mathrm{MS}$ glass vial as a mixed sample for quality control. The extracts were dried in a vacuum concentrator without heat, at $37^{\circ} \mathrm{C}$ for $2 \mathrm{~h}$. A total of $60 \mu 1$ methoxylamine hydrochloride (dissolved in pyridine; final concentration, $20 \mathrm{mg} / \mathrm{ml}$ ) was added to the dried metabolites, which were then incubated at $80^{\circ} \mathrm{C}$ for $20 \mathrm{~min}$ in an oven following mixing and sealing. A total of $80 \mu \mathrm{l}$ BSTFA (containing 1\% TCMS, v/v; Regis Technologies, Inc., Morton Grove, IL, USA) was added to each sample, prior to sealing and further incubation at $70^{\circ} \mathrm{C}$ for $1 \mathrm{~h}$. Subsequently, $5 \mu \mathrm{l}$ FAMEs (standard mixture of fatty acid methyl esters; C8-C16,1 mg/ml; C18-C24,0.5 mg/ml in chloroform; Sigma-Aldrich; Merck $\mathrm{KGaA}$ ) was added to the mixed sample, which was then cooled to room temperature. Each sample was vortex-mixed for $2 \mathrm{~min}$ and carefully transferred to the GC autosampler vials for subsequent GC/TOF-MS analysis.

GC/TOF-MS analysis. GC/TOF-MS analysis was performed using an Agilent 7890 GC system (Agilent Technologies, Inc.) coupled with a Pegasus HT TOF-MS (LECO Corporation, Saint Joseph, MI, USA). The system utilized an Rxi-5Sil MS column (30 m x250 $\mu \mathrm{m}$ inner diameter; $0.25 \mu \mathrm{m}$ film thickness; Restek Corporation, Bellefonte, PA, USA). A $1 \mu$ l aliquot of the analyte was injected in splitless mode. Helium was used as the carrier gas, the front inlet purge flow was $3 \mathrm{ml} / \mathrm{min}$ and the gas flow rate through the column was $20 \mathrm{ml} / \mathrm{min}$. The initial temperature was maintained at $50^{\circ} \mathrm{C}$ for $1 \mathrm{~min}$, which was then raised to $330^{\circ} \mathrm{C}$ at a rate of $10^{\circ} \mathrm{C} / \mathrm{min}$ and maintained at $330^{\circ} \mathrm{C}$ for $5 \mathrm{~min}$. The injection, transfer line and ion source temperatures were 280,280 and $250^{\circ} \mathrm{C}$, respectively. The energy was $-70 \mathrm{eV}$ in electron impact mode. The MS results were acquired in full-scan mode with an m/z range of 30-600 at a rate of 20 spectra per second, following a solvent delay of $366 \mathrm{sec}$.

Data processing and pattern recognition. Chromatogram data acquisition, baseline correction, peak deconvolution, analyte alignment, peak area integration and analyte identification by mass spectral searches based on National Institute of 
Standards and Technology (http://www.nist.gov/index.html) and Fiehn Rtx 5 libraries (LECO Corporation) were performed using the LECO ChromaTOF software version 4.21 (LECO Corporation). All GC-MS data variables were mean-centered and underwent Pareto scaling prior to being subjected to principal component analysis (PCA), partial least squares discriminant analysis (PLS-DA) and orthogonal partial least squares discriminant analysis (OPLS-DA) using SIMCA software version 14.1 (Umetrics; Sartorius AG, Göttingen, Germany). When OPLS-DA was employed, the integrity of the mathematical model was evaluated first, prior to being used for further interpretation. Commonly $\mathrm{R}^{2} \mathrm{Y}$ provides an estimate of how well the model fits the $\mathrm{Y}$ data, whereas $\mathrm{Q}^{2} \mathrm{Y}$ is an estimate of how well the model predicts the Y. When the $\mathrm{Q}^{2} \mathrm{Y}$ and $\mathrm{R}^{2} \mathrm{Y}$ are close to 1 , they indicate an excellent model. The model validity and potential over-fitting of OPLS-DA was checked by performing 200 permutation tests and was visualized using a validation plot.

Determination of most and least effective treatment durations. Blood samples from the right carotid artery of rats were collected at various time-points during reperfusion $(5,15,30$, $45,60,120$ and $180 \mathrm{~min}$ ). The samples were maintained for $30 \mathrm{~min}$ at room temperature and the serum was separated by centrifugation at $2,258 \mathrm{xg}$ for $15 \mathrm{~min}$ at $4^{\circ} \mathrm{C}$. The most and least effective treatment durations were determined by measuring the indicators associated with myocardial injury, CK-MB and LDH. All measurements were performed according to the kit manufacturers' protocols.

Measurement of myocardial apoptosis. The number and distribution of apoptotic cells were detected using an apoptosis In Situ Cell Death Detection kit (Wanleibio, Shenyang, China) according to the manufacturer's protocol. Briefly, myocardial slices from rats in the Sham, Model, SM, DOO and $\mathrm{SM}+\mathrm{DOO}$ groups were stained with DAPI (Sigma; Merck KGaA, Darmstadt, Germany) for the assessment of nuclear morphology. The terminal deoxynucleotidyl transferase-mediated dUTP nick end-labeling (TUNEL)-positive cells were imaged by fluorescence microscopy at x400 magnification (Nikon Corporation, Tokyo, Japan). TUNEL-positive cells were identified by color; the nuclei of the normal myocardium fluoresced blue and the apoptotic cells fluoresced green. The number of apoptotic cells and the total number of cells identified by the double-blind method were used to express the percentage of TUNEL-positive cells.

Evaluation of oxidative stress and inflammation. The activities of SOD and MDA were measured in serum (expressed as $\mathrm{U} / \mathrm{ml}$ or $\mathrm{nmol} / \mathrm{ml}$ of serum) and in cardiac tissue homogenates (expressed as $\mathrm{U} / \mathrm{mg}$ protein or $\mathrm{nmol} / \mathrm{mg}$ protein). The levels of the inflammatory cytokines, TNF- $\alpha$ and IL-6, in the serum samples were quantified using specific ELISA kits for rats. Serum TNF- $\alpha$ and IL-6 levels were calculated using the kit standards and expressed in $\mathrm{pg} / \mathrm{ml}$. All measurements were performed according to the kit manufacturers' protocols.

Statistical analysis. Data are presented as the mean \pm standard deviation of six independent experiments. Statistical analysis was performed using GraphPad Prism software version 6.02
(GraphPad Software, Inc., La Jolla, CA, USA). Differences among groups were analyzed by one-way analysis of variance followed by a post hoc Tukey's multiple comparisons test. $\mathrm{P}<0.05$ was considered to indicate a statistically significant difference.

\section{Results}

Effects of SM and DO on ECG and HWI. When compared with the Sham group, the ST-segment of the Model group was significantly elevated, which demonstrated that the MI/R injury model was successfully established. Pretreatment with Diltiazem, SM, DOA or DOO in MI/R injury rats induced a significant (Diltiazem, SM and DOO, $\mathrm{P}<0.01$; DOA, $\mathrm{P}<0.05$ ) decrease in the ST-segment when compared with the Model group. In addition, the combined treatments of $\mathrm{SM}+\mathrm{DOA}$ and $\mathrm{SM}+\mathrm{DOO}$ produced a greater decrease in the ST-segment, particularly in the SM + DOO group, which induced a significantly lower ST-segment compared with in the SM pretreatment group ( $\mathrm{P}<0.05$; Fig. 1B). As presented in Fig. 1C, heart rate variation in the Model group was significantly higher than that of the Sham group $(\mathrm{P}<0.01)$, the pretreatment groups had no significant difference when compared with Model group, with the exception of the SM + DOO group $(\mathrm{P}<0.05)$, which indicated that only SM + DOO pretreatment had a significant effect on heart rate. Following sacrifice the HWI was measured, and was significantly increased in the Model group compared with in the Sham group $(\mathrm{P}<0.01)$. Rats pretreated with $\mathrm{SM}+\mathrm{DOO}$ exhibited a significant reduction compared with in the Model group ( $\mathrm{P}<0.01$; Fig. 1D).

Effects of SM and DO on myocardial infarct size and myocardial marker enzymes. As presented in Fig. 2A and B, the infarct size of the Model group rat hearts reached $46.91 \pm 2.56 \%$. Conversely, the infarct size decreased to as low as $20.53 \pm 0.98 \%$ in the Diltiazem group, $27.83 \pm 1.70 \%$ in the SM group, $38.59 \pm 1.18 \%$ in the DOA group and $31.55 \pm 2.19 \%$ in the DOO group. In addition, infarct size in the SM + DOA and SM + DOO groups was $25.28 \pm 1.44$ and $20.96 \pm 1.61 \%$, respectively, which was lower than that observed in the singular pretreatment groups. Furthermore, the infarct size was observed to be more effectively decreased in the $\mathrm{SM}+\mathrm{DOO}$ group $(\mathrm{P}<0.01$ vs. Model; $\mathrm{P}<0.01$ vs. $\mathrm{SM})$. As shown in Fig. $2 \mathrm{C}$ and D, when compared with the Sham group, the levels of CK-MB and LDH were significantly increased in the Model group $(\mathrm{P}<0.01)$; however, they were significantly decreased by $\mathrm{SM}$ or $\mathrm{DOO}$ pretreatments $(\mathrm{P}<0.01)$. In addition, pretreatment with SM + DOO further lowered CK-MB and LDH content, which was significantly decreased compared with in the SM group $(\mathrm{P}<0.05)$.

Metabonomics study. Within metabonomics, OPLS-DA approaches are frequently used to distinguish between classes expected to exhibit metabolic differences, each spot represents a sample and each assembly of samples indicates a particular metabolic pattern. In the present study, all samples fell inside the $95 \%$ confidence interval; the samples from all groups were perfectly separated and samples in every group were well clustered. Using the rat serum metabolic profiles obtained in GC/TOF-MS as the basis, the score plot of the OPLS-DA 

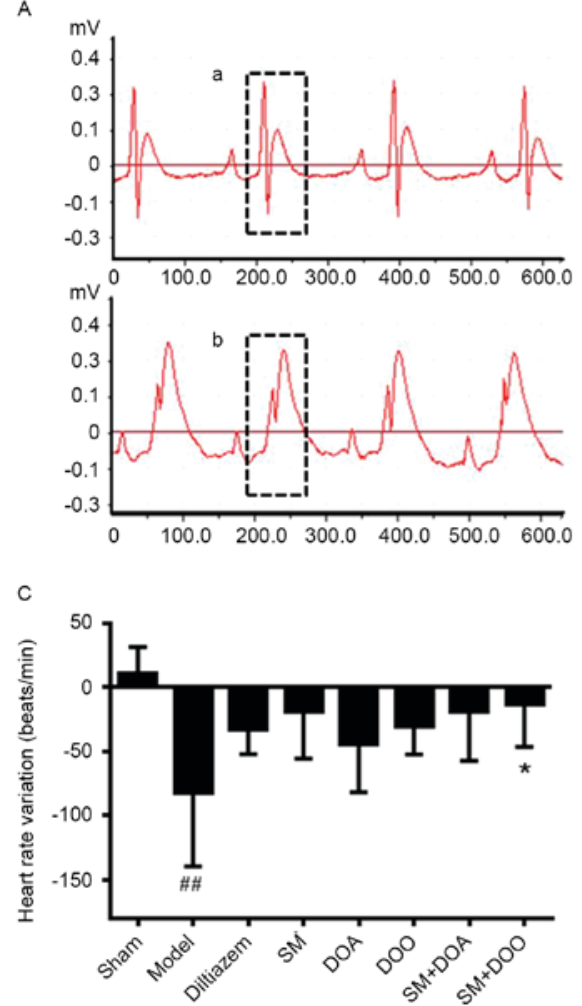

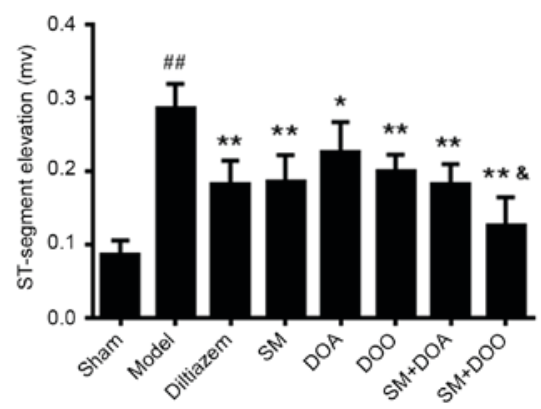

D

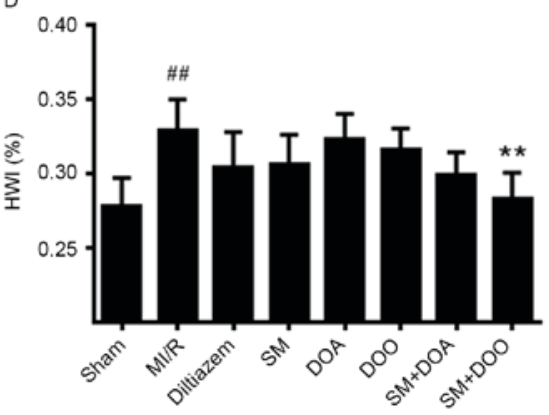

Figure 1. Effects of SM and DO on the ECG results in an ischemia/reperfusion rat model. (A) Representative ECGs (a) before and (b) after coronary artery ligation. (B) ST-segment elevation (mv) in the various groups. (C) Heart rate variation of rats in the various groups. (D) HWI across the various groups, where $\mathrm{HWI}(\%)=\left(\right.$ heart weight/body weight) $\mathrm{x} 100 \%$. All values are presented as the mean \pm standard deviation. ${ }^{\# \#} \mathrm{P}<0.01 \mathrm{vs}$. the Sham group; ${ }^{*} \mathrm{P}<0.05$ and ${ }^{* *} \mathrm{P}<0.01 \mathrm{vs}$. the Model group; ${ }^{\circledR} \mathrm{P}<0.05$ vs. the SM group. SM, Radix Salviae miltiorrhizae; ECG, electrocardiogram; HWI, heart weight index; DOA, aqueous extract of DO; DOO, volatile oil of DO; DO, Lignum Dalbergiae odoriferae.

A

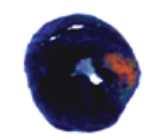

Sham

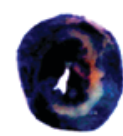

DOA

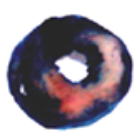

Model

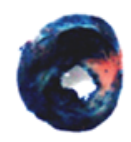

DOO

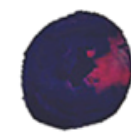

Diltiazem

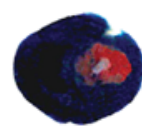

$S M+D O A$

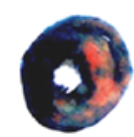

SM

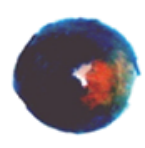

SM+DOO

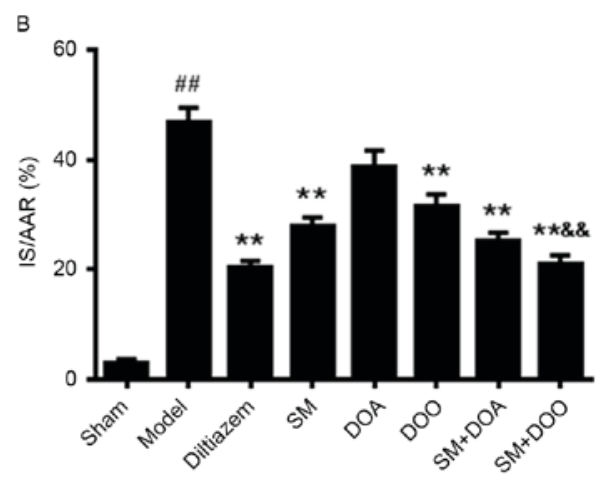

D

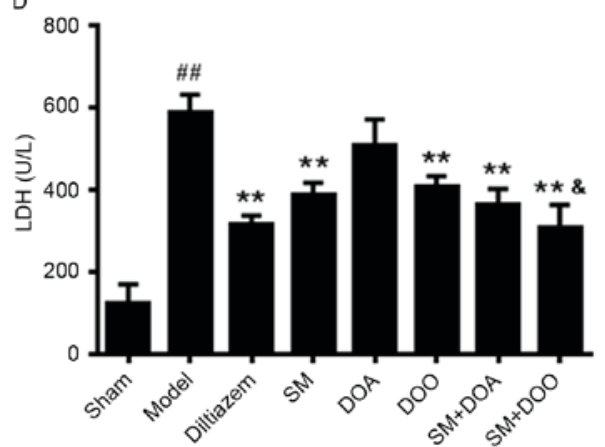

Figure 2. Effects of SM and DO on myocardial damage in rats subjected to myocardial ischemia/reperfusion injury. (A) Representative photographs of heart sections in the various groups. (B) Myocardial infarct area expressed as (IS/AAR)x100\% [IS/AAR(\%)]. (C) Effects of SM and DO on the serum activity of CK-MB in rats. (D) Effects of SM and DO on the serum activity of $\mathrm{LDH}$ in rats. All values are presented as the mean \pm standard deviation. ${ }^{\# \# ~} \mathrm{P}<0.01 \mathrm{vs}$. the Sham group; ${ }^{* *} \mathrm{P}<0.01$ vs. the Model group; ${ }^{\&} \mathrm{P}<0.05$ and ${ }^{\&} \& \mathrm{P}<0.01$ vs. the $\mathrm{SM}$ group. SM, Radix Salviae miltiorrhizae; CK-MB, creatine kinase-MB; LDH, lactate dehydrogenase; DOA, aqueous extract of DO; DOO, volatile oil of DO; DO, Lignum Dalbergiae odoriferae; IS, infarct size; AAR, area at risk. 
A

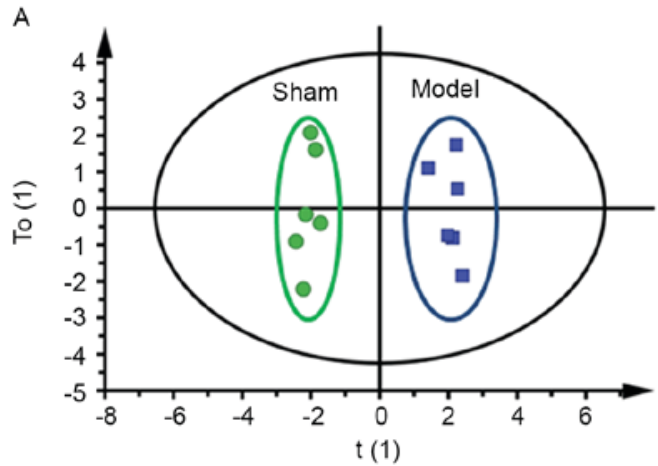

B

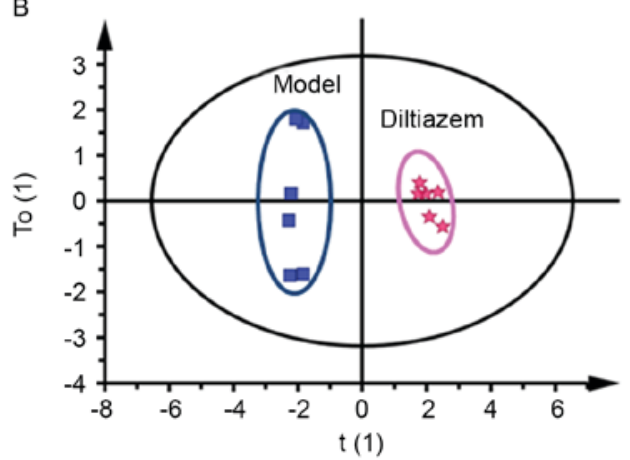

C

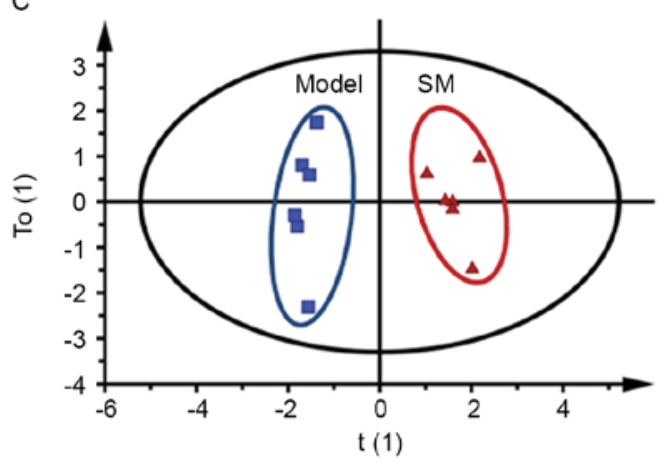

D

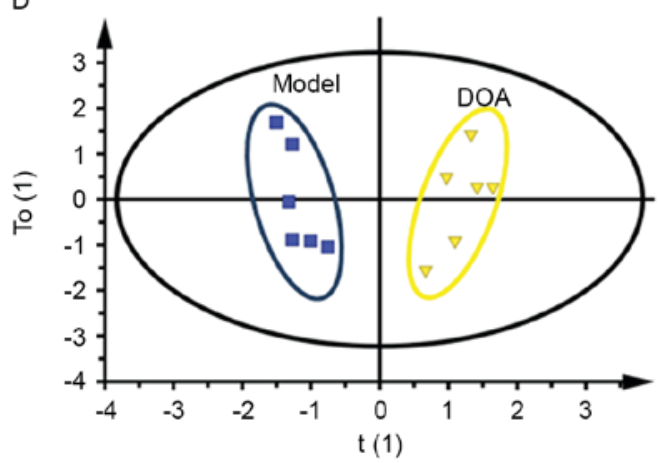

- Sham - Model

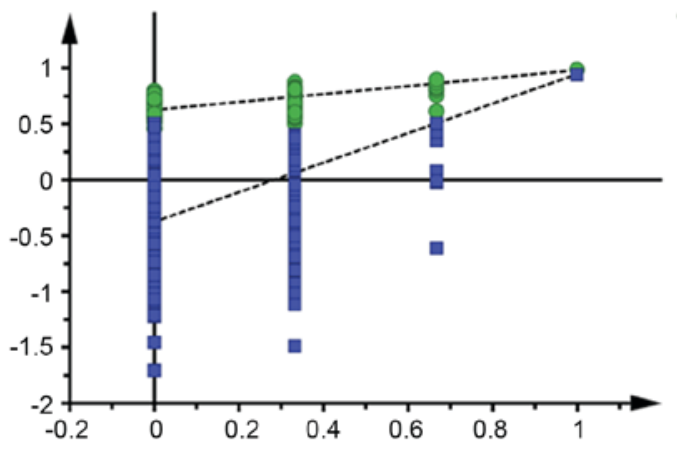

- Model

$\star$ Diltiazem

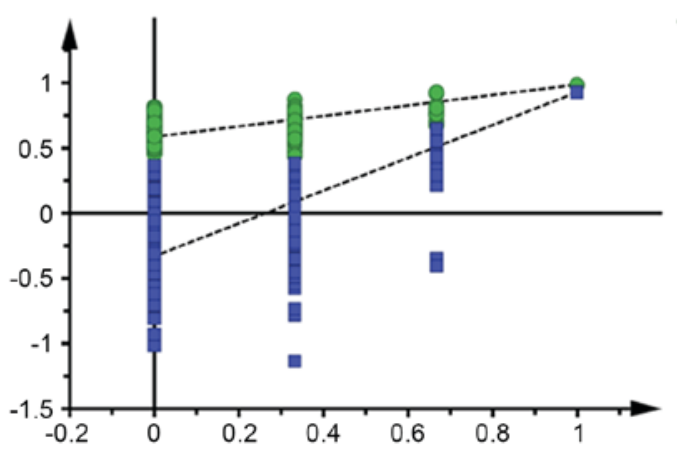

$\begin{aligned} & \mathrm{R}^{2} \\ \text { - } & \mathrm{Q}^{2}\end{aligned}$

- Model

SM

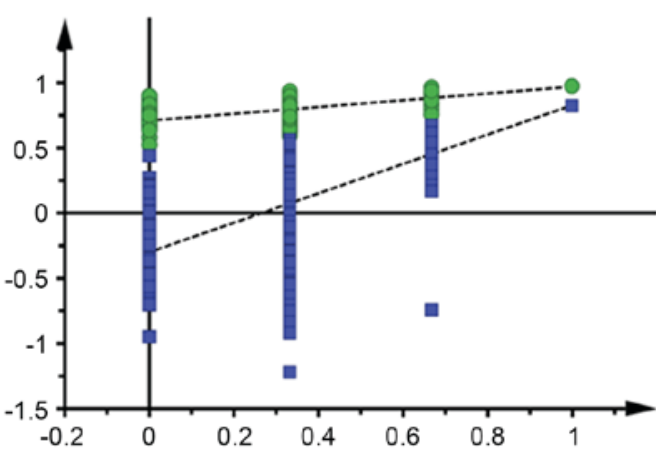

- $R^{2}$

- Model $\checkmark$ DOA

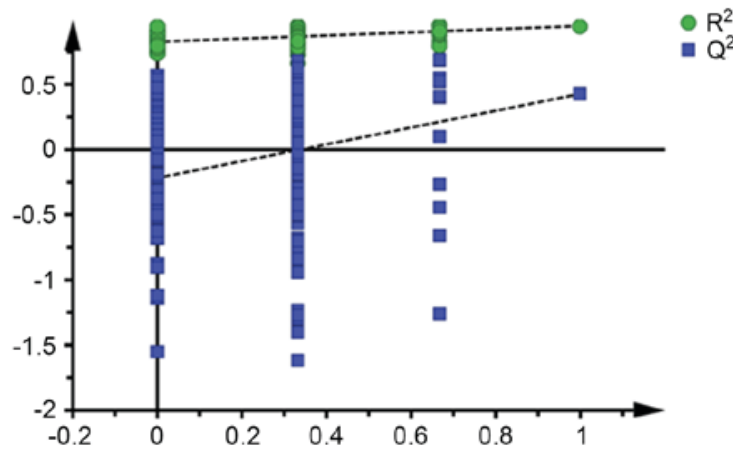

Figure 3. Orthogonal partial least squares discriminant analysis score plots (left-hand column) and the corresponding permutation test ( $\mathrm{n}=200$; right-hand column). (A) Sham group vs. Model group $\left(R^{2} X=0.613, R^{2} Y=0.982, Q^{2} Y=0.947\right)$. (B) Model group vs. Diltiazem group (Positive control group; $R^{2} X=0.587$, $\left.R^{2} Y=0.987, Q^{2} Y=0.929\right)$. (C) Model group vs. $S M$ group $\left(R^{2} X=0.44, R^{2} Y=0.97, Q^{2} Y=0.828\right)$. (D) Model group vs. DOA group $\left(R^{2} X=0.309, R^{2} Y=0.949\right.$, $\left.\mathrm{Q}^{2} \mathrm{Y}=0.427\right)$. SM, Radix Salviae miltiorrhizae; DOA, aqueous extract of DO; DOO, volatile oil of DO; DO, Lignum Dalbergiae odoriferae.

model discriminating the Model group from the Sham group is presented in Fig. 3A $\left(R^{2} \mathrm{X}=0.613, \mathrm{R}^{2} \mathrm{Y}=0.982, \mathrm{Q}^{2} \mathrm{Y}=0.947\right)$, which indicated the success in constructing the MI/R injury rat model. In addition, the Model group was clearly separated from the Diltiazem group $\left(\mathrm{R}^{2} \mathrm{X}=0.587, \mathrm{R}^{2} \mathrm{Y}=0.987\right.$, $\left.\mathrm{Q}^{2} \mathrm{Y}=0.929\right)$, SM group $\left(\mathrm{R}^{2} \mathrm{X}=0.44, \mathrm{R}^{2} \mathrm{Y}=0.97, \mathrm{Q}^{2} \mathrm{Y}=0.828\right)$,
DOA group $\left(R^{2} X=0.309, R^{2} Y=0.949, Q^{2} Y=0.427\right)$, DOO group $\left(R^{2} X=0.372, R^{2} Y=0.95, Q^{2} Y=0.628\right), S M+D O A$ group $\left(R^{2} \mathrm{X}=0.485, \mathrm{R}^{2} \mathrm{Y}=0.994, \mathrm{Q}^{2} \mathrm{Y}=0.927\right)$ and $\mathrm{SM}+\mathrm{DOO}$ group $\left(R^{2} X=0.65, R^{2} Y=0.997, Q^{2} Y=0.957\right.$ ) (Fig. 3B-G). To validate the model, permutation tests with 200 iterations were performed, all permuted $\mathrm{R}^{2}$ (cumulative) and $\mathrm{Q}^{2}$ (cumulative) 

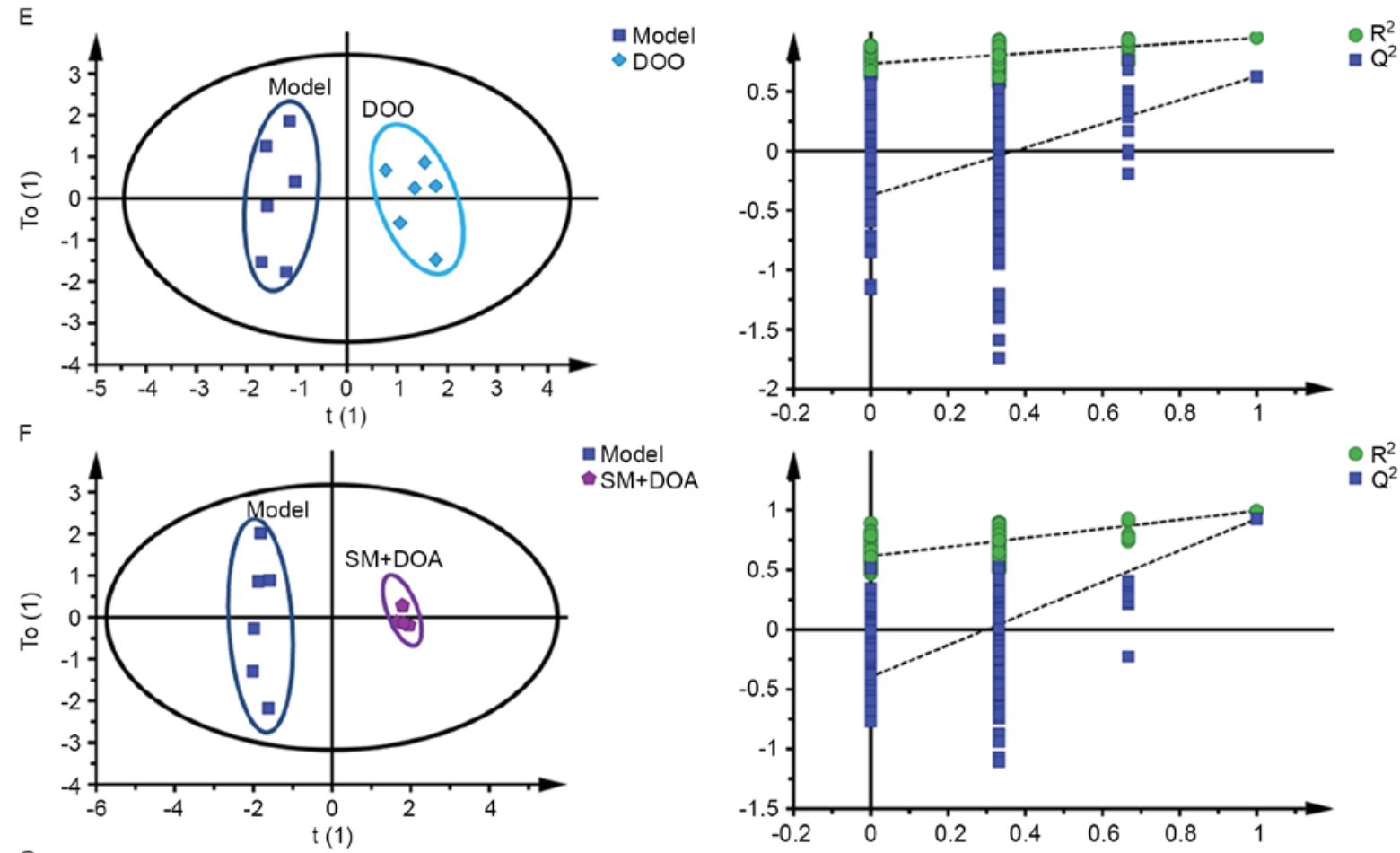

- Model
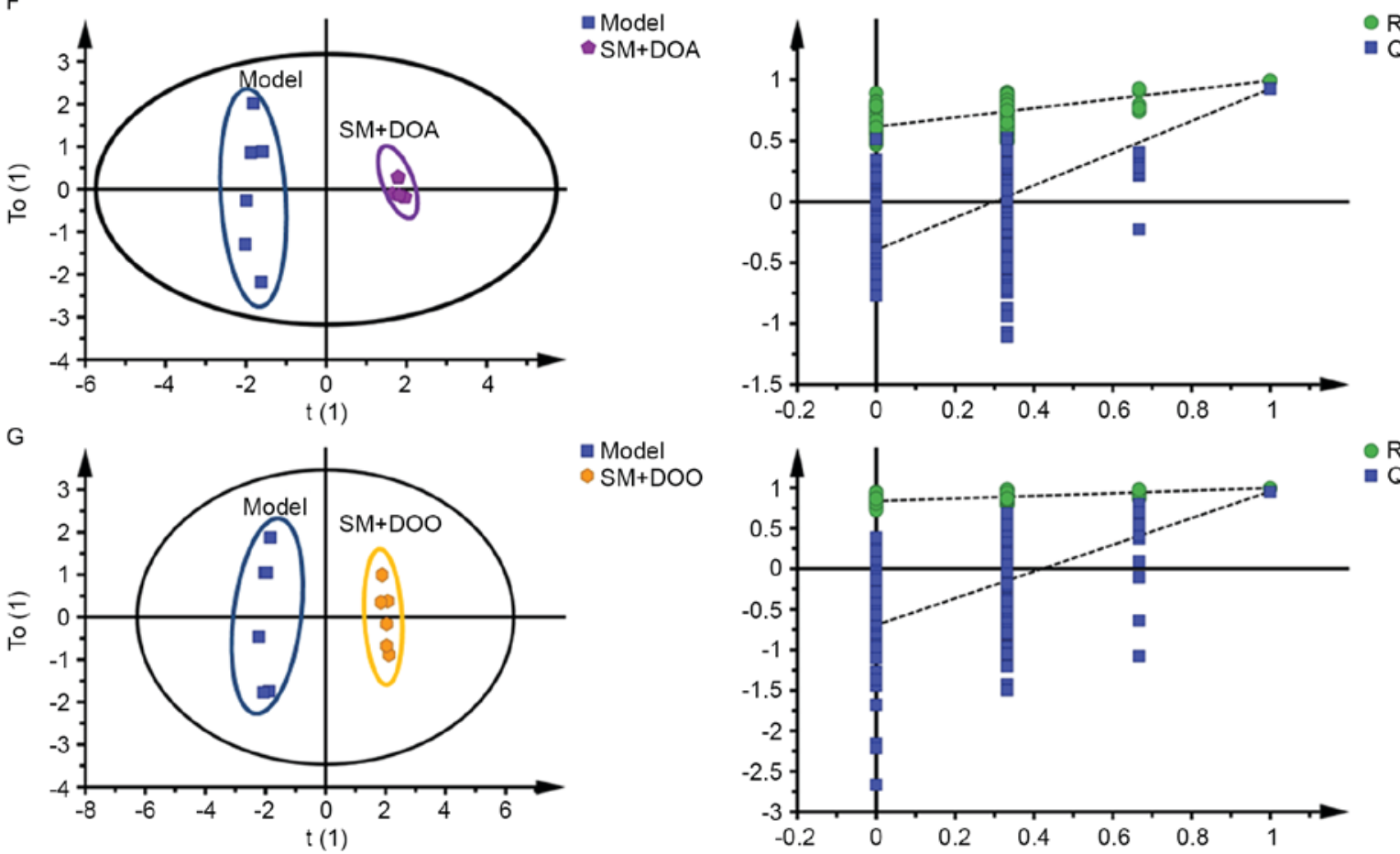

Figure 3. Continued. Orthogonal partial least squares discriminant analysis score plots (left-hand column) and the corresponding permutation test ( $=200$; right-hand column). (E) Model group vs. DOO group $\left(R^{2} X=0.372, R^{2} Y=0.95, Q^{2} Y=0.628\right)$. (F) Model group vs. $S M+D O A$ group $\left(R^{2} X=0.485, R^{2} Y=0.994\right.$, $\left.\mathrm{Q}^{2} \mathrm{Y}=0.927\right)$. (G) Model group vs. $\mathrm{SM}+\mathrm{DOO}$ group $\left(\mathrm{R}^{2} \mathrm{X}=0.65, \mathrm{R}^{2} \mathrm{Y}=0.997, \mathrm{Q}^{2} \mathrm{Y}=0.957\right)$. SM, Radix Salviae miltiorrhizae; DOA, aqueous extract of DO; DOO, volatile oil of DO; DO, Lignum Dalbergiae odoriferae.

values to the left are lower than the original point to the right and the blue regression line of the $\mathrm{Q}^{2}$ (cumulative) points has a negative intercept (Fig. 3 right-hand column), which indicated that the original model was valid.

To evaluate the overall alterations of all endogenous metabolites in the different groups, PCA and PLS-DA approaches were used to distinguish between the groups expected to exhibit metabolic differences. As illustrated in the 2D PCA score plot (Fig. 4A), all of the samples in the Model group were distributed in the right-hand side of the figure and were well separated from those of the Sham and pretreatment groups. When compared to pretreatments with a single herb (SM, DOA or DOO groups), the combination-based pretreatment (SM + DOO group) was much closer to the Sham group and was further away from the Model group. In addition, the distance of the metabolic pattern between the SM + DOO group and the Sham group was shorter than that observed between the Diltiazem group (positive control) and the Sham group. The same results could be seen in the 3D PLS-DA score plot (Fig. 4B).
The most and least effective treatment durations of SM+ DOO. As presented in Fig. 5A and B, the levels of CK-MB and $\mathrm{LDH}$ in the Sham group during reperfusion did not alter greatly over time; however, greater levels were observed in the Model group following $180 \mathrm{~min}$ of reperfusion. The results demonstrated that the most effective treatment duration of $\mathrm{SM}$ was $15 \mathrm{~min}$ and the least effective treatment duration was $180 \mathrm{~min}$. In the DOO group the most effective treatment duration was $45 \mathrm{~min}$ and the least effective treatment duration was $180 \mathrm{~min}$. In the SM + DOO group, the most effective treatment duration was $30 \mathrm{~min}$ and the least effective treatment duration was $180 \mathrm{~min}$. The most and least effective treatment durations were consistent for the levels of CK-MB and LDH.

Anti-apoptotic effects of SM+DOO. Representative photomicrographs revealed that TUNEL-positive cells were more frequently observed in the Model group ( $\mathrm{P}<0.01$ vs. Sham group). Pretreatment with SM, DOO or SM + DOO reduced the percentage of TUNEL-positive cells when compared with 


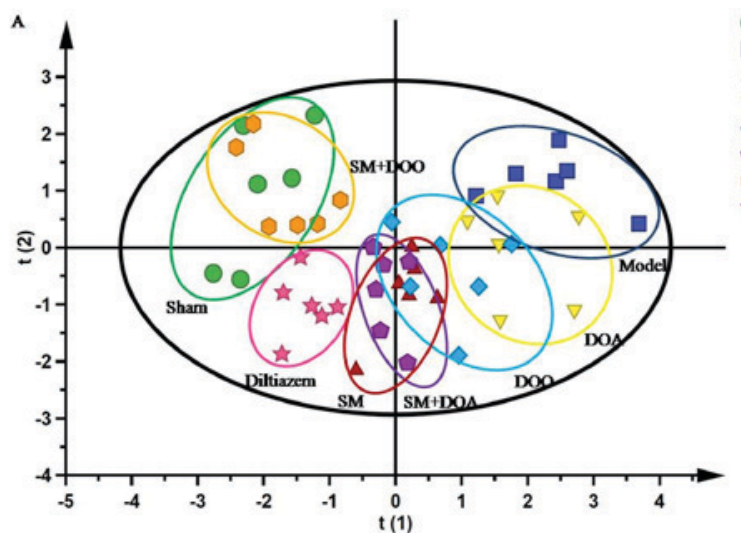

Sham Model DOA $\triangle D O O$ SM+DOA $\mathrm{SM}+\mathrm{DOO}$ Diltiazem

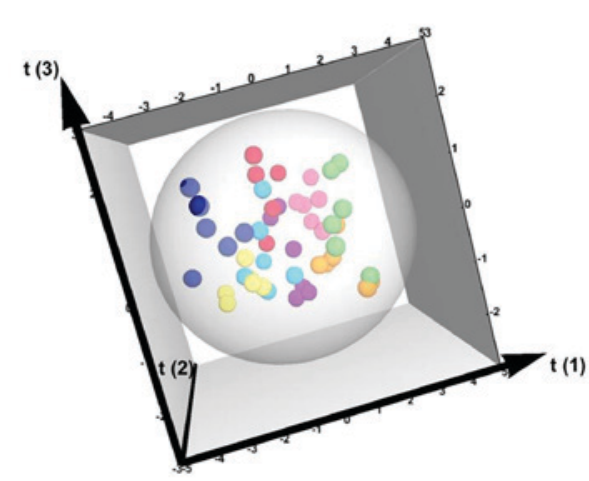

Figure 4. Differential metabolic profile in rat serum among the eight classes (A) 2D principal component analysis score plot. (B) 3D partial least squares discriminant analysis score plot. SM, Radix Salviae miltiorrhizae; DOA, aqueous extract of DO; DOO, volatile oil of DO; DO, Lignum Dalbergiae odoriferae.

the Model group $(32.46 \pm 5.59,39.69 \pm 5.79$ or $16.27 \pm 5.02 \%$ vs. $52.63 \pm 11.02 \% ; \mathrm{P}<0.05)$. The results also revealed a significant difference between the SM and SM + DOO groups $(\mathrm{P}<0.01$; Fig. 6).

Antioxidative and anti-inflammatory effects of $S M+D O O$. As presented in Fig. 7A-D, when compared with the Sham group, the Model group rats exhibited significantly higher levels of MDA and lower SOD activity. The administration of $\mathrm{SM}+\mathrm{DOO}$ reduced MDA content and increased SOD enzymatic activity compared with in the Model group $(\mathrm{P}<0.01)$. In addition, the SM group exhibited decreased MDA content $(\mathrm{P}<0.01)$ and increased SOD activity $(\mathrm{P}<0.05)$ compared with the Model group; however, there was no significance differences between the Model and DOO groups. In addition, there were no significant differences between the SM and $\mathrm{SM}+\mathrm{DOO}$ groups. As shown in Fig. 7E and F, the levels of TNF- $\alpha$ and IL- 6 were significantly elevated in the Model group compared with in the Sham group, and were markedly decreased in the pretreatment groups compared with in the Model group. In addition, the SM + DOO group exhibited a significant decrease in the levels of IL-6 when compared with the SM group $(\mathrm{P}<0.05)$.

\section{Discussion}

In TCM, SM and DO have been commonly used in combination to treat IHD; however, scientific evaluation of their effects

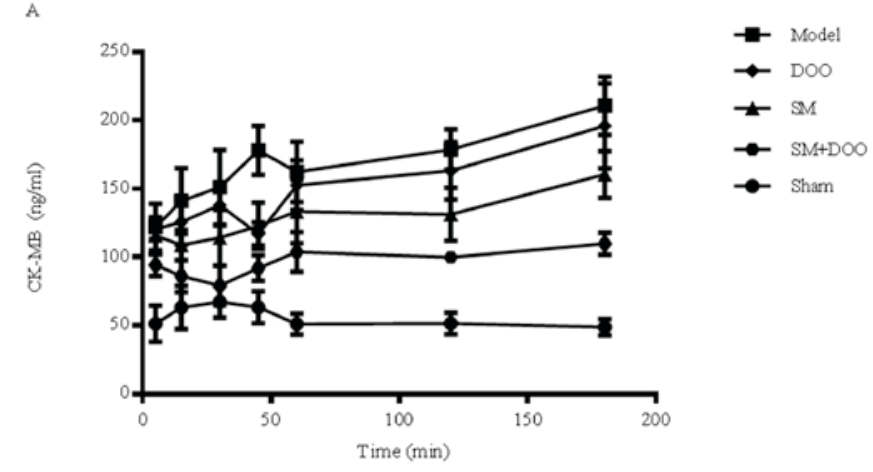

B

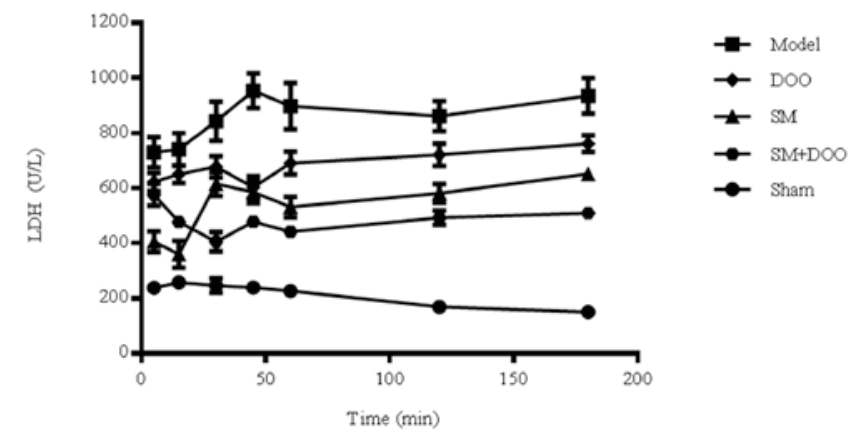

Figure 5. The most and least effective treatment durations of SM, DOO and $\mathrm{SM}+$ DOO during the reperfusion period. (A) Levels of CK-MB at various time-points during reperfusion $(5,15,30,45,60,120$ and $180 \mathrm{~min})$. (B) Levels of $\mathrm{LDH}$ at various time-points during reperfusion $(5,15,30,45,60,120$ and $180 \mathrm{~min})$. All values are presented as the mean \pm standard deviation.SM, Radix Salviae miltiorrhizae; DOO, volatile oil of Lignum Dalbergiae odoriferae; CK-MB, creatine kinase-MB; LDH, lactate dehydrogenase.

and mechanism is lacking. In the present study, a novel strategy for integrating traditional pharmacodynamic evaluation and a metabonomics assay was applied to assess the holistic efficacy and synergistic effects of SM and DO on MI/R injury rats. In addition, the effective combined effects of $\mathrm{SM}+\mathrm{DOO}$ on $\mathrm{MI} / \mathrm{R}$ injury were evaluated in comparison with singular pretreatments and the potential mechanisms were determined.

At present, no single known compound is potent enough to adequately protect the heart against I/R injury. However, combinations of numerous compounds may provide more effective protection against infarction and achieve a greatly improved therapeutic outcome (31). In traditional Chinese prescriptions, SM as a Jun herb for diseases has the primary therapeutic effect, and DO as the Shi herb enhances the primary therapeutic effect of SM by exerting the function of guiding the main bioactive compounds to the site of the disease, and adjusting the potency of the herbs in order to function synergistically (29). In the present study, SM and DO combinations exerted greater significant effects on cardioprotection compared with SM, DOA or DOO singular pretreatments, particularly the combined treatment of SM and DOO. SM + DOO exhibited a greater potential to improve ECG results and heart rate, reduce HWI and myocardial infarct size, and decrease the levels of CK-MB and LDH. When compared with the Diltiazem group (positive control group), $\mathrm{SM}+$ DOO treatment performed just as well. From a chemical point of view, the reason why SM + DOO have a unique effect in the body may be due to the two groups of 

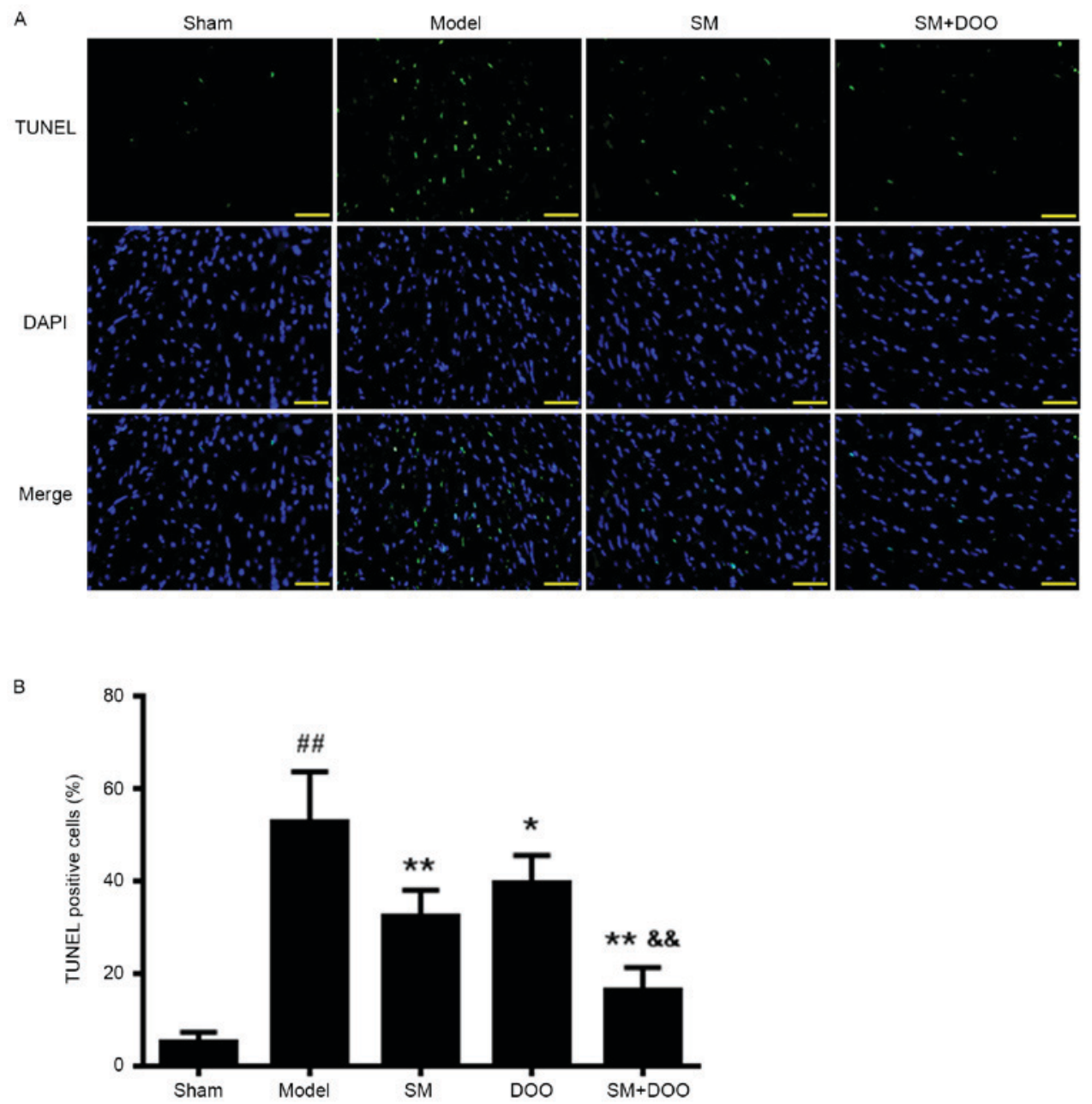

Figure 6. Anti-apoptotic effects of SM, DOO and SM + DOO on rats subjected to myocardial ischemia/reperfusion injury. (A) Representative images of apoptotic cells stained green with TUNEL in ischemic cardiac tissue. Total nuclei were stained blue with DAPI. (B) Quantitative analysis of apoptotic rate. TUNEL-positive cells (\%) are expressed as (number of apoptotic myocytes/total myocytes)x100\%. Scale bar, $50 \mu \mathrm{m}$. All values are presented as the mean \pm standard deviation. ${ }^{\# \#} \mathrm{P}<0.01$ vs. the Sham group; ${ }^{*} \mathrm{P}<0.05$ and ${ }^{* *} \mathrm{P}<0.01$ vs. the Model group; ${ }^{\text {\&\&}} \mathrm{P}<0.01$ vs. the SM group. SM, Radix Salviae miltiorrhizae; DOO, volatile oil of Lignum Dalbergiae odoriferae; TUNEL, terminal deoxynucleotidyl transferase-mediated dUTP nick end-labeling.

active ingredients interacting to produce a synergistic effect that targets numerous sites, thereby generating a greater therapeutic effect.

The release of myocardial enzymes is considered to be an important indicator of the degree of myocardial damage (32). At present, detection of the serum levels of CK-MB and LDH in patients with acute myocardial ischemia or infarction may facilitate early diagnosis (33). In the present study, following myocardial cell damage, the serum CK-MB and LDH levels were significantly increased, whereas in all of the pretreatment groups, with the exception of the DOA group, the serum levels of CK-MB and LDH were reduced in the rats, which may be associated with stability of the myocardial cell membrane and integrity of myocardial cells. In addition, the SM + DOO group had a lower CK-MB and LDH content, which was significantly reduced compared with in the SM group. It is possible that DOO may guide the main bioactive compounds of SM to target the disease site or it may improve the effectiveness of the treatment. Further analysis revealed that the least effective treatment duration of SM, DOO and SM + DOO was 180 min during the reperfusion period, and the most effective treatment durations were as follows: SM, $15 \mathrm{~min}$; DOO, $45 \mathrm{~min}$;
$\mathrm{SM}+\mathrm{DOO}, 30 \mathrm{~min}$. These findings suggested that DOO may serve a role in myocardial protection and may also enhance the therapeutic effects of SM.

Metabonomics, with its impressive and ever-increasing coverage of endogenous compounds and its intrinsic high-throughput capacity, has been employed in numerous areas. Analysis of the overall metabolites of biological samples reveals the therapeutic potential of TCM agents. The results of OPLS-DA indicated that the MI/R injury rat model was successfully established and all of the pretreatment groups had a marked level of efficacy in MI/R injury rats. The global metabolic profiles of the rats from the eight groups were subjected to PCA and PLS-DA to compare the metabolic effects of SM and DO on endogenous metabolites. All the samples in the Model group were separated from those of the Sham group, which demonstrated that the MI/R injury model was successfully established. The results also suggested that the SM, DOA and DOO treatments exerted efficacy in MI/R injury rats, and the combination of SM and DOO increased the therapeutic efficacy. The distance of the metabolic pattern between the SM + DOO and Sham groups was much shorter than that observed between the Diltiazem (positive control) and 
A

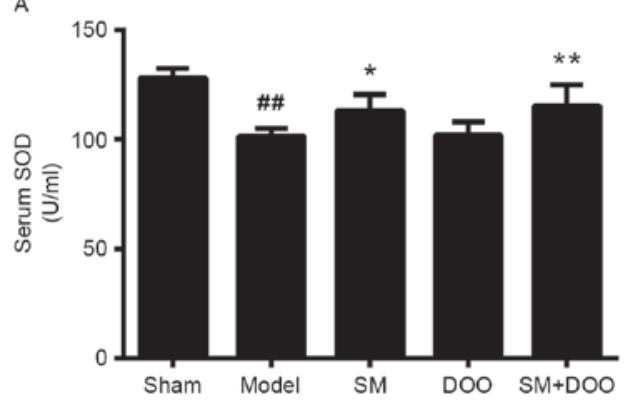

$\mathrm{C}$
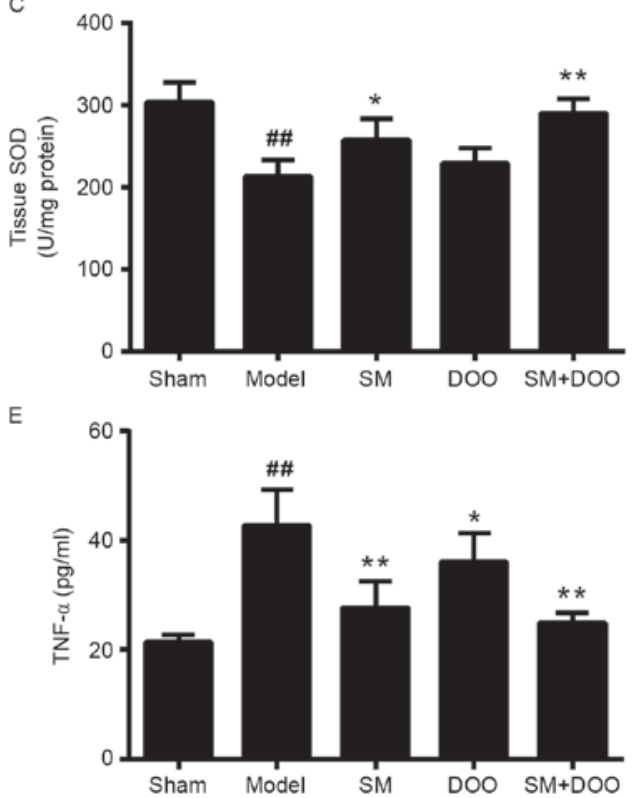
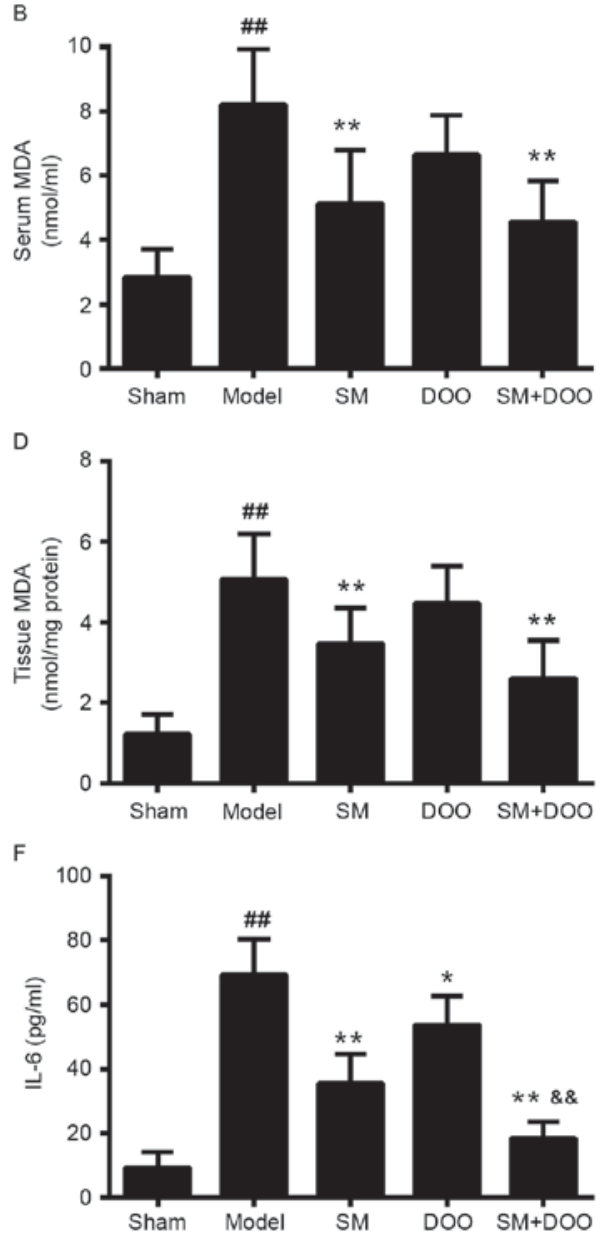

Figure 7. Antioxidative and anti-inflammatory effects of SM, DOO and SM + DOO in rats subjected to myocardial ischemia/reperfusion injury. (A) Serum SOD activities. (B) Serum MDA content. (C) Tissue SOD activities. (D) Tissue MDA content. (E) Serum TNF- $\alpha$ levels. (F) Serum IL-6 levels. All values are presented as the mean \pm standard deviation. ${ }^{\# \#} \mathrm{P}<0.01$ vs. the Sham group; ${ }^{*} \mathrm{P}<0.05$, and ${ }^{* *} \mathrm{P}<0.01$ vs. the Model group; \&\& $<<0.01$ vs. the $\mathrm{SM}$ group. $\mathrm{SM}$, Radix Salviae miltiorrhizae; DOO, volatile oil of Lignum Dalbergiae odoriferae; SOD, superoxide dismutase; MDA, malondialdehyde; TNF- $\alpha$, tumor necrosis factor- $\alpha$; IL-6, interleukin-6.

Sham groups, which indicated that the metabolic pattern of the $\mathrm{SM}+\mathrm{DOO}$ group rats was more comparable to healthy rats than the Diltiazem group. This outcome was consistent with the aforementioned results, which may be due to differences in the underlying mechanisms of SM + DOO and Diltiazem. It is possible that $\mathrm{SM}+\mathrm{DOO}$ consists of a complex mixture of compounds and thus treats diseases by acting on numerous targets. From a metabonomic perspective, the effects of the combination group (SM + DOO) were more marked than those of the other groups and the result is in accordance with the experimental pharmacodynamic results.

The results revealed that $5 \mathrm{~g} / \mathrm{kg} \mathrm{SM}$ combined with $0.5 \mathrm{~g} / \mathrm{kg}$ DOO produced potent cardioprotective effects when compared with the individual treatments and other combinations. The present study investigated the potential mechanism of SM + DOO. Apoptosis serves an important role in reperfusion injury (34). Recent studies have demonstrated that ischemic-induced apoptosis and necrosis contribute to autophagic cardiomyocyte death and cardiomyocyte loss in myocardial ischemic injury $(35,36)$. In the present study, SM, DOO and SM + DOO reduced the number of TUNEL-positive cells and the anti-apoptotic effects displayed by the $\mathrm{SM}+\mathrm{DOO}$ group were markedly greater than those of SM alone. These results indicated that DOO may contribute to SM-induced inhibition of apoptosis and reduction in myocardial damage following MI/R injury.

The generation of oxygen free radicals and the subsequent formation of reactive oxygen species, which may induce lipid peroxidation and oxidative stress, have been implicated as major causative factors of myocardial injury $(37,38)$. SOD is an antioxidant that protects the myocardium from peroxidation (39). MDA is a toxic end product of lipid peroxidation, and MDA levels can directly indicate the rate and extent of lipid peroxidation, and indirectly reveal the capacity for eliminating free radicals (40). The present study demonstrated that $\mathrm{SM}+$ DOO enhances the ability to eliminate oxygen free radicals and reduces lipid peroxidation injury of the cell membrane and myocardial injury. Inflammation has also been recognized as a major factor in ischemic progression, and an increasing body of evidence has demonstrated that enhanced levels of inflammatory markers are associated with ischemia (41-43). In the present study, SM, DOO and SM + DOO decreased the levels of TNF- $\alpha$ and IL- 6 in serum, and the anti-inflammatory effects of $\mathrm{SM}+\mathrm{DOO}$ were markedly greater compared with SM 
treatment alone when evaluating IL-6 levels. These findings suggested that DOO may contribute to the anti-inflammatory action of SM.

Current anti-ischemic medications, including $\beta$-blockers, calcium channel blockers, nitroglycerin and angiotensin inhibitors (44), are limited by their hemodynamic side effects due to their potent single target action. In addition, MI/R injury is caused by numerous factors; due to the complex multifactorial nature of the disease, modern pharmacotherapies using single target drugs are usually not effective. A combination therapy, such as $\mathrm{SM}+\mathrm{DOO}$, is urgently required as it may have significant potential in treating MI/R injury.

In conclusion, the present study demonstrated that a specific dosage of SM + DOO $(5 \mathrm{~g} / \mathrm{kg} \mathrm{SM}$ and $0.5 \mathrm{ml} / \mathrm{kg}$ DOO) exerted a notable cardioprotective effect on infarct size and cardiac function recovery when compared with the respective individual treatments. This effect may be achieved partly through reducing myocardial cell apoptosis, oxidative stress and the inflammatory cascade, based on their pleiotropic pharmacological properties. In addition, the most effective treatment durations of SM, DOO and SM + DOO were 15, 45 and $30 \mathrm{~min}$, respectively, whereas the least effective duration for all three treatments was $180 \mathrm{~min}$. However, the clinical therapeutic effects of the combined treatment and the mechanisms underlying its cardioprotective effect still require further investigation.

\section{Acknowledgements}

The present study was supported by the National Natural Science Foundation of China (grant no. 81470174). The present study was presented at the BIT 8th Annual International Congress of Cardiology (China, 2016) and the abstract was published in the Journal of the American College of Cardiology (45).

\section{References}

1. Forman MB and Jackson EK: Myocardial reperfusion injury. $\mathrm{N}$ Engl J Med 357: 2408-2410, 2007.

2. Sivaraman V and Yellon DM: Pharmacologic therapy that simulates conditioning for cardiac ischemic/reperfusion injury. J Cardiovasc Pharmacol Ther 19: 83-96, 2014.

3. Monassier JP: Reperfusion injury in acute myocardial infarction. From bench to cath lab. Part I: Basic considerations. Arch Cardiovasc Dis 101: 491-500, 2008.

4. Rodriguez-Porcel M, Zhu XY, Chade AR, Amores-Arriaga B, Caplice NM, Ritman EL, Lerman A and Lerman LO: Functional and structural remodeling of the myocardial microvasculature in early experimental hypertension. Am J Physiol Heart Circ Physiol 290: H978-H984, 2006.

5. Liu H, Shang J, Chu F, Li A, Wu B, Xie X, Liu W, Yang H and Tong T: Protective effects of Shen-Yuan-Dan, a traditional Chinese medicine, against myocardial ischemia/reperfusion injury in vivo and in vitro. Evid Based Complement Alternat Med 2013: 956397, 2013.

6. Lakshmi SV, Padmaja G, Kuppusamy P and Kutala VK: Oxidative stress in cardiovascular disease. Indian J Biochem Biophys 46: 421-440, 2009.

7. Gottlieb RA: Cell death pathways in acute ischemia/reperfusion injury. J Cardiovasc Pharmacol Ther 16: 233-238, 2011.

8. Koenitzer JR and Freeman BA: Redox signaling in inflammation: Interactions of endogenous electrophiles and mitochondria in cardiovascular disease. Ann NY Acad Sci 1203: 45-52, 2010.

9. Li J,Zhang $\mathrm{H}$ and Zhang $\mathrm{C}$ : Role of inflammation in the regulation of coronary blood flow in ischemia and reperfusion: Mechanisms and therapeutic implications. J Mol Cell Cardiol 52: 865-872, 2012
10. Guan Y, Yin Y, Zhu YR, Guo C, Wei G, Duan JL, Wang YH, Zhou D, Quan W, Weng Y, et al: Dissection of mechanisms of a Chinese medicinal formula: Danhong injection therapy for myocardial ischemia/reperfusion injury in vivo and in vitro. Evid Based Complement Alternat Med 2013: 972370, 2013.

11. Zhao N, Liu YY, Wang F, Hu BH, Sun K, Chang X, Pan CS, Fan JY, Wei XH, Li X, et al: Cardiotonic pills, a compound Chinese medicine, protects ischemia-reperfusion-induced microcirculatory disturbance and myocardial damage in rats. Am J Physiol Heart Circ Physiol 298: H1166-H1176, 2010.

12. Liu Q, Li J, Wang J, Li J, Janicki JS and Fan D: Effects and mechanisms of Chinese herbal medicine in ameliorating myocardial ischemia-reperfusion injury. Evid Based Complement Alternat Med 2013: 925625, 2013.

13. Geng Z, Huang L, Song M and Song Y: Protective effect of a polysaccharide from Salvia miltiorrhiza on isoproterenol (ISO)-induced myocardial injury in rats. Carbohyd Polym 132: 638-642, 2015 .

14. Sugiyama A, Zhu BM, Takahara A, Satoh Y and Hashimoto K: Cardiac effects of Salvia miltiorrhiza/Dalbergia odorifera mixture, an intravenously applicable Chinese medicine widely used for patients with ischemic heart disease in China. Circ J 66 : $182-184,2002$

15. Zhao L, Gao H, Zhao Y and Lin D: Metabonomic analysis of the therapeutic effect of Zhibai Dihuang pill in treatment of streptozotocin-induced diabetic nephropathy. J Ethnopharmacol 142: 647-656, 2012.

16. Liu P and Wang P: Application of metabolomics technology in the research of Chinese medicine. Chin J Integr Med 20: 307-310, 2014.

17. Chen M, Su M, Zhao L, Jiang J, Liu P, Cheng J, Lai Y, Liu Y and Jia W: Metabonomic study of aristolochic acid-induced nephrotoxicity in rats. J Proteome Res 5: 995-1002, 2006.

18. Griffin JL and Bollard ME: Metabonomics: Its potential as a tool in toxicology for safety assessment and data integration. Curr Drug Metab 5: 389-398, 2004.

19. Yin P, Zhao X, Li Q, Wang J, Li J and Xu G: Metabonomics study of intestinal fistulas based on ultraperformance liquid chromatography coupled with Q-TOF mass spectrometry (UPLC/Q-TOF MS). J Proteome Res 5: 2135-2143, 2006.

20. Ganna A, Salihovic S, Sundstrüm J, Broeckling CD, Hedman AK, Magnusson PK, Pedersen NL, Larsson A, Siegbahn A, Zilmer M, et al: Large-scale metabolomic profiling identifies novel biomarkers for incident coronary heart disease. PLoS Genet 10: e1004801, 2014.

21. Luo L, Zhen L, Xu Y, Yang Y, Feng S, Wang S and Liang S: (1)H NMR-based metabonomics revealed protective effect of Naodesheng bioactive extract on ischemic stroke rats. J Ethnopharmacol 186: 257-269, 2016.

22. Chang KL and Ho PC: Gas chromatography time-of-flight mass spectrometry (GC-TOF-MS)-based metabolomics for comparison of caffeinated and decaffeinated coffee and its implications for Alzheimer's disease. PLoS One 9: e104621, 2014.

23. Klein J, Papadopoulos T, Mischak H and Mullen W: Comparison of CE-MS/MS and LC-MS/MS sequencing demonstrates significant complementarity in natural peptide identification in human urine. Electrophoresis 35: 1060-1064, 2014.

24. Tang YM, Wang JP, Bao WM, Yang JH, Ma LK, Yang J, Chen H, $\mathrm{Xu} \mathrm{Y}$, Yang LH, Li W, et al: Urine and serum metabolomic profiling reveals that bile acids and carnitine may be potential biomarkers of primary biliary cirrhosis. Int J Mol Med 36: 377-385, 2015.

25. Koek MM, Muilwijk B, van der Werf MJ and Hankemeier T: Microbial metabolomics with gas chromatography/mass spectrometry. Anal Chem 78: 1272-1281, 2006.

26. Wang SX, Luo K, Liang J, Fan F, Li H, Zheng JB and Zheng XH: Metabolomics study on the synergistic interaction between Salvia miltiorrhiza and Lignum Dalbergiae odoriferae used as 'Jun-Shi' herbs in a S. miltiorrhiza recipe. Med Chem Res 20: $16-22,2011$

27. Liu R, Sun J, Bi K and Guo D: Identification and determination of major flavonoids in rat serum by HPLC-UV and HPLC-MS methods following oral administration of Dalbergia odorifera extract. J Chromatogr B Analyt Technol Biomed Life Sci 829: 35-44, 2005.

28. Yin Y, Guan Y, Duan J, Wei G, Zhu Y, Quan W, Guo C, Zhou D, Wang Y, Xi M and Wen A: Cardioprotective effect of Danshensu against myocardial ischemia/reperfusion injury and inhibits apoptosis of H9c2 cardiomyocytes via Akt and ERK1/2 phosphorylation. Eur J Pharmacol 699: 219-226, 2013. 
29. Zheng X, Zhao X, Wang S, Luo K, Wei Y and Zheng J: Co-administration of Dalbergia odorifera increased bioavailability of Salvia miltiorrhizae in rabbits. Am J Chin Med 35: 831-840, 2007.

30. Quan W, Wei G, Zhou D, Zhu Y, Guo C, Wang Y, Weng Y, Xi M and Wen A: Magnesium lithospermate B reduces myocardial ischemia/reperfusion injury in rats. Pharm Biol 51: 1355-1362, 2013.

31. Deng Y, Yang M, Xu F, Zhang Q, Zhao Q, Yu H, Li D, Zhang G, Lu A, Cho K, et al: Combined salvianolic acid B and ginsenoside Rg1 exerts cardioprotection against ischemia/reperfusion injury in rats. PLoS One 10: e135435, 2015.

32. Arya DS, Bansal P, Ojha SK, Nandave M, Mohanty I and Gupta SK: Pyruvate provides cardioprotection in the experimental model of myocardial ischemic reperfusion injury. Life Sci 79: 38-44, 2006.

33. Kemp M, Donovan J, Higham H and Hooper J: Biochemical markers of myocardial injury. Br J Anaesth 93: 63-73, 2004.

34. Yan J, Duan J, Wu X, Guo C, Yin Y, Zhu Y, Hu T, Wei G, Wen A and Xi M: Total saponins from Aralia taibaiensis protect against myocardial ischemia/reperfusion injury through AMPK pathway. Int J Mol Med 36: 1538-1546, 2015.

35. Tao Z, Chen B, Tan X, Zhao Y, Wang L, Zhu T, Cao K, Yang Z, Kan YW and Su H: Coexpression of VEGF and angiopoietin-1 promotes angiogenesis and cardiomyocyte proliferation reduces apoptosis in porcine myocardial infarction (MI) heart. Proc Natl Acad Sci USA 108: 2064-2069, 2011.

36. Kung G, Konstantinidis K and Kitsis RN: Programmed necrosis, not apoptosis, in the heart. Circ Res: 1017-1036, 2011.

37. Garciarena CD, Fantinelli JC, Caldiz CI, Chiappe de Cingolani G, Ennis IL, Pérez NG, Cingolani HE and Mosca SM: Myocardial reperfusion injury: Reactive oxygen species vs. NHE-1 reactivation. Cell Physiol Biochem 27: 13-22, 2011.

38. Jaeschke $\mathrm{H}$ and Woolbright BL: Current strategies to minimize hepatic ischemia-reperfusion injury by targeting reactive oxygen species. Transplant Rev (Orlando) 26: 103-114, 2012.
39. Zheng W, Huang LZ, Zhao L, Wang B, Xu HB, Wang GY, Wang ZL and Zhou H: Superoxide dismutase activity and malondialdehyde level in plasma and morphological evaluation of acute severe hemorrhagic shock in rats. Am J Emerg Med 26: 54-58, 2008.

40. Schettler V, Methe H, Staschinsky D, Schuff-Werner P, Müller GA and Wieland E: Review: The oxidant/antioxidant balance during regular low density lipoprotein apheresis. Ther Apher 3: 219-226, 1999.

41. Willerson JT and Ridker PM: Inflammation as a cardiovascular risk factor. Circulation 109 (21 Suppl 1): II2-II10, 2004.

42. Gasparyan AY: Cardiovascular risk and inflammation: Pathophysiological mechanisms, drug design, and targets. Curr Pharm Des 18: 1447-1449, 2012.

43. Song F, Li H, Sun J and Wang S: Protective effects of cinnamic acid and cinnamic aldehyde on isoproterenol-induced acute myocardial ischemia in rats. J Ethnopharmacol 150: 125-130, 2013.

44. Yan B, Deng Y, Hou J, Bi Q, Yang M, Jiang B, Liu X, Wu W and Guo D: UHPLC-LTQ-Orbitrap MS combined with spike-in method for plasma metabonomics analysis of acute myocardial ischemia rats and pretreatment effect of Danqi Tongmai tablet. Mol Biosyst 11: 486-496, 2015.

45. Fei M, Duan J and Xi M: GW27-e0912 cardioprotective effects of Radix Salviae miltiorrhizae and Lignum Dalbergiae odoriferae on rat myocardial ischemia/reperfusion injury. J Am Coll Cardiol (Suppl S 68): C64, 2016. 